\section{mCRPC: $\alpha$-Strahler verbessert auch Lebensqualität}

Der $\alpha$-Strahler Radium-223 (Ra-223) verbesserte in der Studie ALSYMPCA bei Männern mit kastrationsresistentem Prostatakarzinom (CRPC) und Knochenmetastasen das Gesamtüberleben. Nun gibt es Daten zur Lebensqualität.

$E_{\text {inent }}$ ne Behandlung mit Ra-223 wird Patienten mit CRPC und symptomatischen Knochenmetastasen vor sowie nach der Docetaxel-Therapie empfohlen. Grundlage dafür ist der Überlebensvorteil, der in der ALSYMPCA-Studie mit dem $\alpha$-Strahler erreicht wurde. In der Studie hatten 921 Patienten randomisiert Ra-223 ( $\mathrm{n}=614)$ oder Placebo $(\mathrm{n}=$ 307) erhalten, jeweils in Kombination mit der Standardversorgung.

Nun wurden die Daten zur gesundheitsbezogenen Lebensqualität ausgewertet, die mithilfe des Fragebogens EuroQoL 5D (EQ-5D) und des FACT-Prostate ermittelt wurden. In der Ra-223Gruppe kam es bei signifikant mehr Patienten zu einer bedeutsamen Verbesserung des EQ-5D-Scores als in der Pla- cebogruppe (29,2 vs. $18,5 \%$; $\mathrm{p}=0,004$ Odds Ratio [OR] 1,82).

Ähnliche Ergebnisse zeigten sich beim OR 1,70). Analysen der FACT-P-Subskalen zeigten, dass die in der Ra-223Gruppe erzielten Verbesserungen vor allem in 3 von 5 Subskalen erreicht wurden, nämlich im emotionalen und funktionalen Wohlbefinden sowie bei den Prostatakrebssymptomen.

Der Anteil an Patienten mit relevanter Verschlechterung im EQ-5D-Score war unter Ra-223 signifikant niedriger als unter Placebo (36 vs. $54 \%$; $p<0,001$; OR 0,48). Beim FACT-P-Score war der Anteil an Patienten mit einer relevanten Verschlechterung unter Ra-223 numerisch, aber nicht signifikant niedriger als FACT-P-Score (24,6 vs. 16,1\%; $\mathrm{p}=0,020$; unter Placebo (44,3 vs. 51,6\%; $\mathrm{p}=0,095$; OR 0,75).

Darüber hinaus war die Therapie mit Ra-223 mit einem höheren EQ-5D-Score im Vergleich zu Placebo assoziiert $(0,56$ vs. 0,$50 ; p=0,002)$. Die Ergebnisse zum FACT-P-Score waren ähnlich $(99,08$ vs. $95,22 ; \mathrm{p}=0,004)$ und zeigten eine signifikant geringere Abnahme seit Studienbeginn unter Radium-223 ( $\mathrm{p}=0,004)$.

Die vorherige Anwendung von Docetaxel und die gleichzeitige Gabe eines Bisphosphonats hatte keinen signifikanten Einfluss auf die Ergebnisse zur Lebensqualität.

Fazit: Die ALSYMPCA-Studie zeigt, dass die Therapie mit dem $\alpha$-Strahler Ra-223 bei Patienten mit CRPC und Knochenmetastasen nicht nur das Gesamtüberleben verlängert, sondern auch die Lebensqualität signifikant verbessert.

Judith Neumaier

Nilsson S et al. Patient-reported quality-of-life analysis of radium-223 dichloride from the phase III ALSYMPCA study. Ann Oncol. 2016; 27(5):868-74.

\title{
Prostatakarzinom: Mit der Androgendeprivation sofort beginnen
}

\begin{abstract}
Eine Androgendeprivationstherapie (ADT) eignet sich für Männer mit einem Prostatakarzinom, die nach kurativer Therapie einen PSA-Anstieg aufweisen oder die für eine kurative Therapie nicht geeignet sind. Unklar ist aber, welches der optimale Zeitpunkt für den Beginn der ADT ist.
\end{abstract}

empfohlenen zeitlichen Abstand von $>2$ Jahren nach Randomisierung). Es konnte jegliche Art der Androgendeprivation verordnet werden. Nach median 5 Jahren waren in der Gruppe mit sofortiger ADT $11 \%$ der Männer verstorben, in der Gruppe mit verzögerter ADT $20 \%$. Dies spiegelte sich auch im Gesamtüberleben (OS) wider (5-Jahres OS: 91,2 vs. $86,4 \%$, 7-Jahres-OS: 81,0 vs. $65,5 \%$; log-rank $\mathrm{p}=0,047)$. Die nicht adjustierte Hazard Ratio für das Gesamtüberleben (sofortige vs. verzögerte Androgendeprivation) betrug $0,55(\mathrm{p}=$ $0,050)$. Außerdem war die Zeit bis zur lokalen Progression oder Entwicklung von Fernmetastasen nach sofortiger Androgendeprivation signifikant länger.
Die häufigsten schwerwiegenden unerwünschten Ereignisse waren kardiovaskuläre Ereignisse Sie traten bei $9 \%$ der Patienten mit sofortiger und bei $6 \%$ mit verzögerter $\mathrm{ADT}$ auf.

Fazit: Die sofortige ADT verbesserte das Gesamtüberleben signifikant im Vergleich zur verzögerten ADT bei Männern, die einen PSA-Anstieg nach der kurativen Therapie aufwiesen oder für die kurative Therapie ungeeignet waren. Gillian M Duchesne und Kollegen werten die Ergebnisse als neuenMaßstab für die Überlebens- und Morbiditätsraten, die mit den Patienten in Zusammenhang mit ihren Therapieoptionen diskutiert werden sollten. Judith Neumaier

Duchesne GM et al. Timing of androgendeprivation therapy in patients with prostate cancer with a rising PSA (TROG 03.06 and VCOG PR 01-03 [TOAD]): a randomised, multicentre, non-blinded, phase 3 trial. Lancet Oncol. 2016; 17(6):727-37. 